\title{
Management of Osteogenesis Imperfecta: A Multidisciplinary Comprehensive Approach
}

\author{
Tae-Joon Cho, MD, Jung Min Ko, MD*, Hyoungmin Kim, MD, \\ Hyung-Ik Shin, $\mathrm{MD}^{\dagger}$, Won Joon Yoo, MD, Chang Ho Shin, MD \\ Division of Pediatric Orthopaedics, Seoul National University Children's Hospital, Seoul National University College of Medicine, Seoul, \\ ${ }^{*}$ Department of Pediatrics, Seoul National University Children's Hospital, Seoul National University College of Medicine, Seoul, \\ ${ }^{\dagger}$ Department of Rehabilitation Medicine, Seoul National University Children's Hospital, Seoul National University College of Medicine, Seoul, Korea
}

Osteogenesis imperfecta (OI) is characterized by recurring fractures and limb and spine deformities. With the advent of medical therapeutics and the discovery of causative genes, as well as the introduction of a newly devised intramedullary rod, the general condition and ambulatory function of patients diagnosed with $\mathrm{Ol}$ have been improved over the past decades. This review covers recent developments in research and management of 0 .

Keywords: Osteogenesis imperfecta, Gene, Bisphosphonate, Intramedullary nailing, Scoliosis

Osteogenesis imperfecta (OI) is a heterogeneous group of genetic skeletal disorders characterized by bone fragility and low bone mass, which manifests as multiple fractures and deformities of the spine and extremities.

\section{GENETICS}

OI is mostly inherited as an autosomal dominant trait; however, some cases are autosomal recessive. Mutations in type I collagen genes (COL1A1 and COL1A2) were found to be the most common causes of OI in $70 \%-80 \%$ of all cases, characterized by an autosomal dominant inheritance. Type $\mathrm{V}$ is the other autosomal dominant OI subtype, which is caused by a single recurrent mutation of IFITM5 $(\sim 10 \%) .{ }^{1,2)}$ To date, more than 10 OI genes of autosomal recessive trait have been identified (10\%-15\%). The 2019 version of the nosology and classification system of genetic skeletal disorders listed 17 genes under the name of OI. ${ }^{3)}$ Most of them are involved in the biosynthesis of type I collagen (COL1A1, COL1A2, CRTAP, LEPR1, PPIB,

Received March 23, 2020; Accepted September 28, 2020

Correspondence to: Tae-Joon Cho, MD

Division of Pediatric Orthopaedics, Seoul National University Children's Hospital, 101 Daehak-ro, Jongno-gu, Seoul 03080, Korea

Tel: +82-2-2072-1863, Fax: +82-2-764-2718

E-mail: tjcho@snu.ac.kr
SERPINH1, FKBP10, and BMP1) or differentiation and function of osteoblasts (SP7, TMEM38B, WNT1, CRE$B 3 L 1$, and $S P A R C)$. However, the function of some genes responsible for the OI phenotype (IFITM5, SERPINF1, and TENT5A) has yet to be elucidated. Genetic testing is indicated when a clinical diagnosis is not obvious or when molecular confirmation is legally required such as differentiation from child abuse, exemption of military service, and so on. Because those having mutation of WNT1 or SERPINF1 are known to be less responsive to bisphosphonate treatment than other subtypes, ${ }^{4,5)}$ genotyping could be helpful in determining medical treatment.

Considering numerous causative genes and their phenotypic similarity, it is more appropriate to test all the known OI genes simultaneously. Many of the OI genes such as COL1A1 and COL1A2 are huge in size and expensive to analyze via Sanger sequencing. Therefore, exome sequencing or target sequencing using panels of known OI genes via next-generation sequencing technology is the diagnostic test of choice for OI mutations. However, these methods cannot be used to detect large copy number variations including intragenic exonal deletions or duplications, which is an important limitation.

The authors have conducted molecular genetic analysis using next-generation sequencing and/or Sanger sequencing in 177 Korean patients with OI and found that 96 patients (54\%) carried COL1A1 mutations, 39 (22\%) 
COL1A2, 10 (6\%) IFITM5, 5 (3\%) FKBP10, 1 BMP1, and 1 WNT1, and no mutation was found in 25 patients (14\%) (unpublished data). Thus, $88 \%$ of all confirmed mutations involve type I collagen genes, compared with $6.6 \%$ of $I F$ ITM5 (type V) and $4.6 \%$ of autosomal recessive cases in our cohort.

\section{CLASSIFICATION}

Various systems of clinical classification have been proposed for OI. In the past, those who sustained fracture(s) before or at the time of birth were grouped under "OI congenita," and the others were included under "OI tarda." The latter was further classified into "OI tarda gravis" if the first fracture occurred before 1 year of age, or "OI tarda levis" if after 1 year of age. ${ }^{6}$ Sillence classification ${ }^{7)}$ was introduced in 1979 and remains as the most widely used classification. Type I is the mildest form of OI with distinctly blue sclera, remaining intensely blue throughout life. Well-managed patients of this type are usually community ambulators without walking aids and enjoy sports activity to some extent. The patients' stature is usually below average compared with peers or genetic target heights estimated in unaffected parents although still within normal limits in most cases. Type II patients are the most severely affected, who succumb during the prenatal or perinatal period of respiratory distress due to extreme fragility of the ribs and pulmonary hypoplasia or malformation or hemorrhage of the central nervous system. Hence, they do not present to orthopaedic surgeons. Type III patients are the most severely affected survivors with blue sclera, who present with numerous, almost uncountable fractures from birth, frequently leading to severe limb deformity if not aggressively managed. Severe spinal deformity develops frequently from the second decade. Patients show profound short stature and are usually wheelchair-bound in adulthood. Type IV patients are characterized by normal color of the sclerae and intermediate severity of bone fragility. They usually remain as community ambulators in adulthood, but need to use walking aids in most cases. Presence or absence of dentinogenesis imperfecta is used to subclassify the groups into A and B. Dental problems including dentinogenesis imperfecta or prognathism are common and could be disabling, which require a dental care by specialists of OI.

The clinical subtypes are not clearly demarcated, and patients are at different degrees of the continuous disease spectrum of OI encompassing types 1 to 4 . In the year 2000, a characteristic clinical phenotype of OI, distinct from types I to IV, was introduced. The patients may have been classified as type IV because of normal white sclera, moderate but diverse bone fragility and fractures, and frequent scoliosis. However, they showed distinct characteristics of ectopic ossification, including ossification of the forearm interosseous membrane, hyperplastic callus formation, intermittent painful subperiosteal bone formation, and heterotopic ossification of the muscles and fasciae, which resulted in bony ankyloses of the joints. This type was denoted as type $\mathrm{V}$ and comprised approximately $10 \%$ of OI patients. Radial head dislocation is a common manifestation in all types of OI, but is almost always present in type $\mathrm{V}^{8-10)}$

Identification of multiple causative genes for OI has led to a genetic classification into specific types. ${ }^{11)}$ However, because the difference in clinical phenotypes is not apparent among patients with different causative genes, the clinical classification based on the 4 types of Sillence classification in addition to type $\mathrm{V}$ is currently used clinically, and the recent version of the nosology and classification system of genetic skeletal disorders is based on this paradigm. ${ }^{3)}$

\section{MEDICAL TREATMENT}

Vitamin D and calcium are vital components of the skeleton, and their deficiency can aggravate osteopenia caused by genetic defects in OI. ${ }^{12)}$ Decreased outdoor activity due to limited mobility makes the OI patients susceptible to vitamin $\mathrm{D}$ deficiency. However, it is important to prevent their deficiency, without overdosing, which can induce hypervitaminosis D, hypercalciuria, and subsequent nephrocalcinosis.

After the epoch-making report of bisphosphonate usage in the medical management of $\mathrm{OI},{ }^{13)}$ cyclic administration of pamidronate (a second-generation bisphosphonate) and subsequent application of other bisphosphonates have become the gold standard for the treatment of $\mathrm{OI}^{1{ }^{14}}$ Bisphosphonate is incorporated into the newly formed bone matrix and is released from the bone matrix by osteoclastic activity. Subsequently, it suppresses osteoclasts and prevents bone matrix resorption by osteoclasts. As most OI patients are in a high bone turnover state, keeping excessive bone matrix is an advantage, even though it is still defective. Bisphosphonate therapy was effective for patients with moderate-to-severe OI during the growth period. The more severe the condition and younger the patients, the more effective was the therapy. Bisphosphonate administration has been shown to increase bone mineral density, leaving sclerotic changes on radiographs called zebra lines (Fig. 1), decreased apprehension and risk of 
recurrent fractures, and improved mobility of the patients. Several studies reported decreased fracture frequency with bisphosphonate therapy, but some did not. ${ }^{14)}$ Regardless of the results of fracture frequency, the therapy must be considered beneficial if the fracture frequency remains the

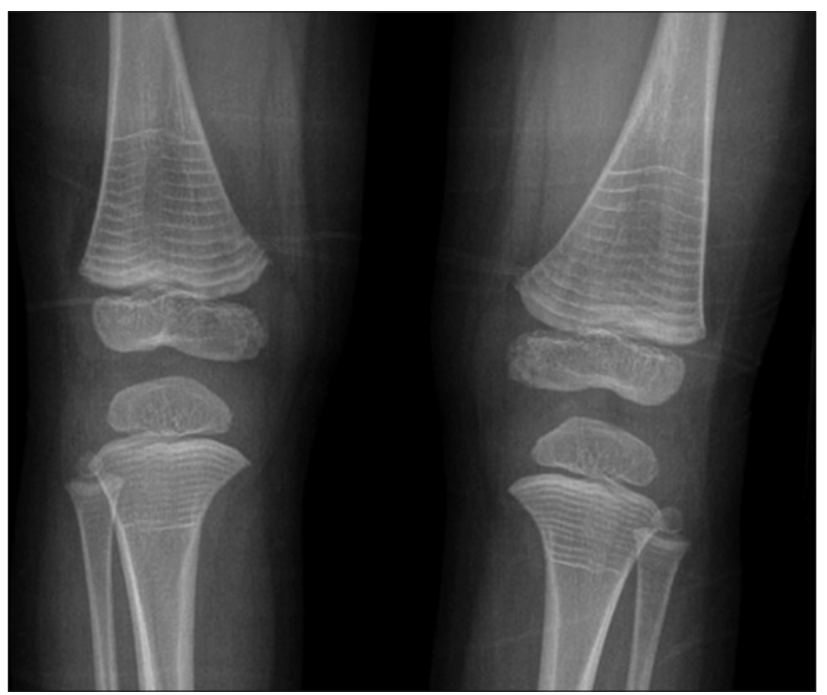

Fig. 1. Zebra lines at the metaphyses of long bones are formed by cyclic administration of pamidronate. Osteosclerotic lines parallel to the physis represent the primary spongiosa produced during each cycle of pamidronate infusion. same with improved patients' mobility. However, it does not seem to prevent the development or progression of scoliosis. ${ }^{15-17)}$

Many types of bisphosphonates (intravenous: pamidronate, zoledronate, and ibandronate; oral: alendronate, risedronate, and ibandronate) have been used to manage OI ${ }^{18)}$ Immediate side effects of bisphosphonates include hypocalcemia and flu-like symptoms, which need to be paid attention to, especially at the first infusion. Prolonged use or overdose of bisphosphonate interferes with skeletal remodeling and increases the risk of obliteration of the marrow cavity, which is not conducive for intramedullary (IM) rod installation, delays healing of osteotomy sites, ${ }^{19}$ and predisposes to atypical fractures at the proximal femur. Moreover, the pharmaceutical effect plateaus 2 to 3 years after initiation. Bisphosphonate therapy can be reduced by halving the dosage or doubling the interval and by monitoring bone density improvement. The authors' protocol is summarized in Fig. 2.

Other medications are currently under clinical trial. Denosumab, a RANKL-blocking antibody, is one of the promising agents under phase 3 clinical trials in adult patients with OI. It improved bone density and mechanical properties and decreased fracture frequency in an OI mouse model. ${ }^{20)}$ A clinical trial of OI patients with SERPINF1 mutations, who do not respond to bisphosphonate treatment, also showed promising results. Sclerostin-

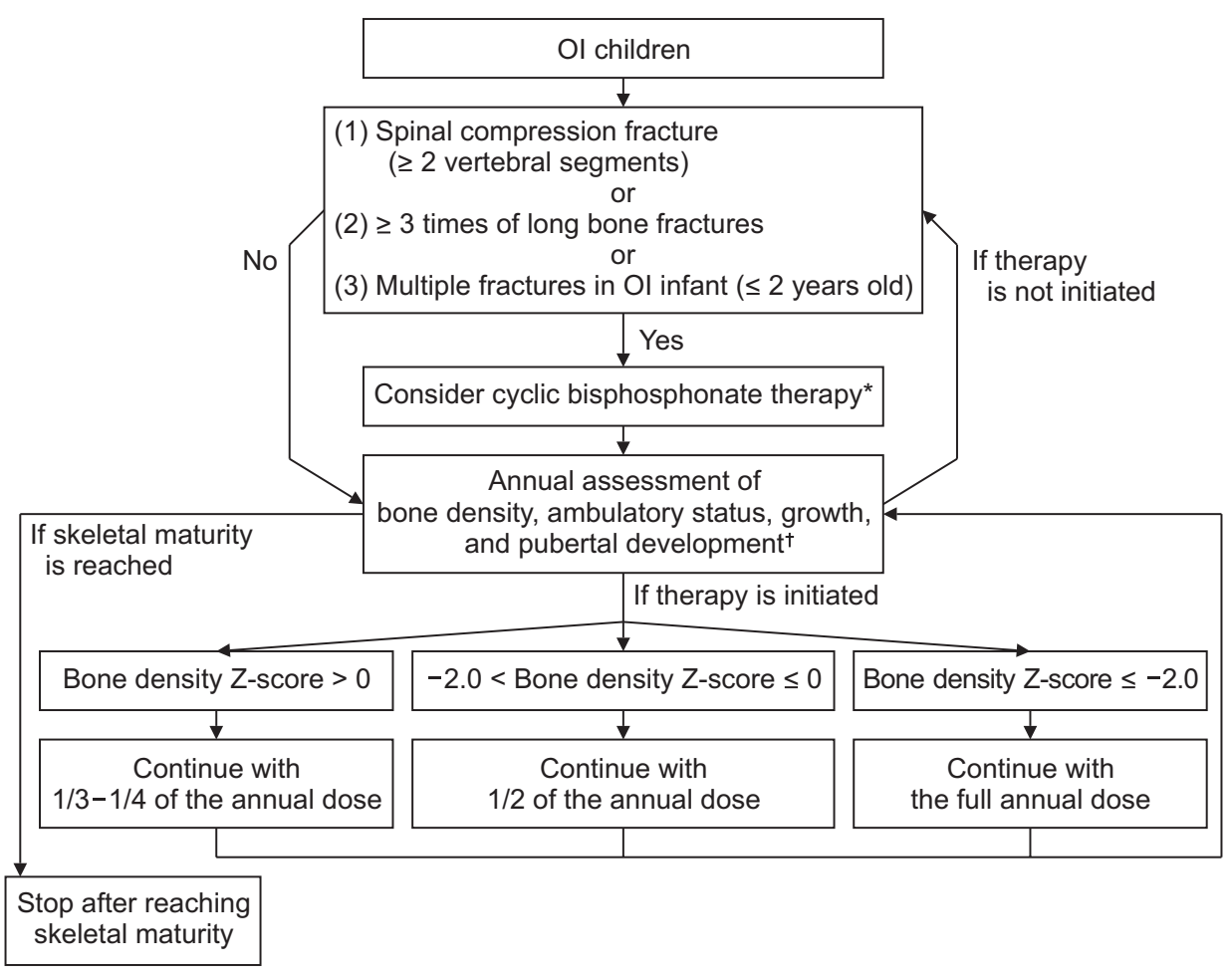

Fig. 2. Protocol outlining cyclic bisphosphonate therapy for osteogenesis imperfecta (OI) patients. *If cyclic bisphosphonate therapy is decided, starting with the full annual dose is recommended. Pamidronate: $9 \mathrm{mg} / \mathrm{kg} / \mathrm{yr}, 4-9$ divided doses (max $60 \mathrm{mg} / \mathrm{dose}$ ). Zoledronate: $0.1 \mathrm{mg} / \mathrm{kg} / \mathrm{yr}$, 2 divided doses (max $5 \mathrm{mg} / \mathrm{yr}$ ). If bone density Z-score is $>2.0$, running or sprinting is possible, normal height of vertebrae is preserved, or $0-2$ times of long bone fractures occur per year, initiation of cyclic bisphosphonate therapy can be delayed with annual monitoring. 
blocking antibody targeting $\mathrm{WNT} / \beta$-catenin signaling demonstrated anabolic effects in an OI mouse model ${ }^{21}$ and is expected to be effective in WNT-related OI in adults. A parathyroid hormone (PTH) analogue used for osteoporosis in a limited way may be tried for adult patients with OI. ${ }^{22)}$ However, the PTH analogue is not approved for treatment of children, due to its risk of osteosarcoma identified in animal studies. A phase 1 clinical trial of anti-transforming growth factor- $\beta$ antibody for OI was recently initiated based on results showing inhibition of osteoclast formation. ${ }^{23)}$ Recombinant human growth hormone failed to show a pharmacological effect in decreasing fracture frequency; however, some researchers have proposed a combination therapy with bisphosphonate to promote height gain in children with OI, although this has yet to be validated. ${ }^{24)}$ Bone marrow transplantation designed to introduce healthy bone-forming cells ${ }^{25}$ ) does not appear to be satisfactory when weighed against its risk. Gene targeting or editing approaches may be the ultimate treatment for this genetic disorder, but there have been only proof-of-concept studies so far.

\section{PHYSICAL THERAPY AND ORTHOTICS}

Physical therapy plays a critical role in the care of patients with OI to maximize function. ${ }^{26)}$ Inability to accomplish daily activities without specialized equipment can compromise safety. For children with severe OI, parents may be advised to "protect" their children with OI by carrying them on pillows and avoiding recreational activities. However, this well-intentioned approach did not protect children from fractures and may have prevented development and functional independence. Parents should understand that gaining of bone strength depends on muscle pull ${ }^{27)}$ as well as weight-bearing through standing, walking, and lifting. Therefore, at a minimum, a passive standing program using frames should be tried. It is important to provide the appropriate mobility aids such as a posterior walker, a walker with trunk/pelvis support and canes (axilla/ forearm crutches or tripod/single-point canes) depending on the severity of the condition. Walkers may contain seats and/or forearm platforms to disperse upper extremity weight-bearing. ${ }^{28)}$ Braces may be used to protect long bones, especially the tibia. A patellar tendon-bearing total contact brace with hinged ankle (Fig. 3) may be useful during postoperative or post-fracture rehabilitation period.

Physiotherapy includes muscle strengthening, aerobic exercise, and recreational pursuits. The specific exercise program depends on the person's age, level of func- tion, severity of OI, and needs and desires. Having fun and feeling a sense of accomplishment are additional goals in any exercise program. Treatment should not be confined to "therapy hours" only. Very short exercise efforts during the day, as short as 5 minutes, will often result in improvement more quickly than an hour-long session once or twice a week.

Children with OI can excel in the water, particularly if the activity is presented as recreational and independent exploration. Walking in the water may be possible for individuals who are unable to walk in the air. Water activities in childhood can be the foundation for a lifelong, enjoyable fitness activity. ${ }^{29)}$ Adults with OI can benefit from water activity as well. It is an excellent form of aerobic conditioning and may enhance skeletal strength. However, because water activities alone have little impact on gaining of bone strength, adults should incorporate other weightbearing exercises such as walking.

\section{FRACTURE MANAGEMENT}

Frequency and risk of fracture depend upon not only the severity of bone stock deficiency, but also deformity of the long bones, age and character of the patient, and family, as well as community environment. Minor injuries, which would not break bones in normal subjects, may induce fractures in OI patients, and in such conditions, the fracture fragments may not displace substantially. In early childhood, conservative treatment is commonly used. Increased attention should be paid to preventing angular or rotational malunion. In neonatal or infantile cases of se-

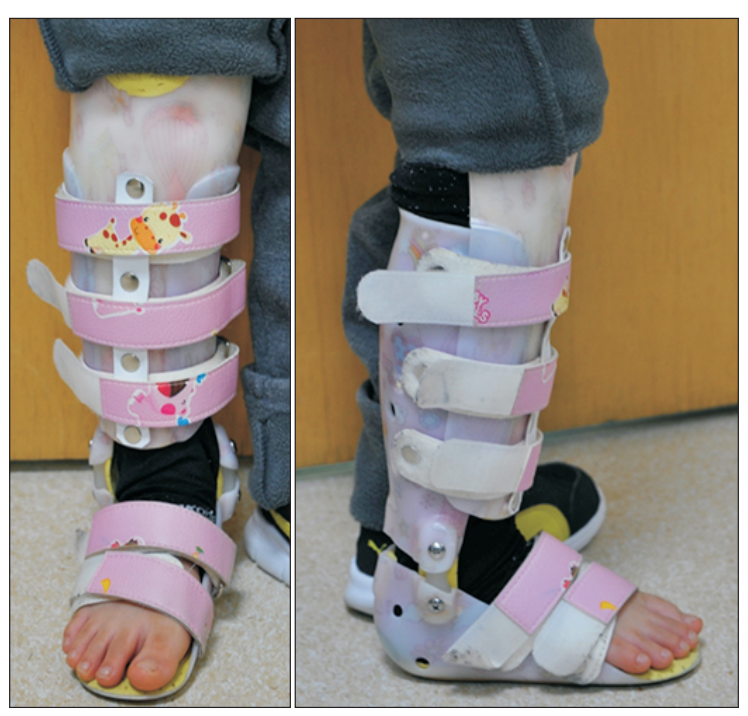

Fig. 3. Patellar tendon-bearing total contact brace with hinged ankle. 
vere OI, fractures of the femur shaft occur frequently, and Pavlik harness is preferred to the long leg splint to prevent anterior angulation malunion. Prolonged immobilization in the cast or splint may cause disuse osteopenia, aggravating the preexisting osteopenic condition. However, the callus formed across the fracture site also contains a defective matrix as in other parts of the bone and is biomechanically less strong than radiographically estimated. Considering these 2 contradictory conditions, the conservatively treated fracture should be mobilized as soon as possible but protected long term using a functional brace. To protect a tibial fracture, the authors use a total contact, patellar tendon-bearing, a short leg brace with hinged ankle (Fig. 3). However, as for the femur, a functional brace is useful only for the distal femoral lesion and may be detrimental for the diaphysis or proximal femoral fractures because the proximal end of the brace may increase the stress on the lesion.

Surgical fixation for long bone fractures is indicated at a younger age in OI patients than in normal population,
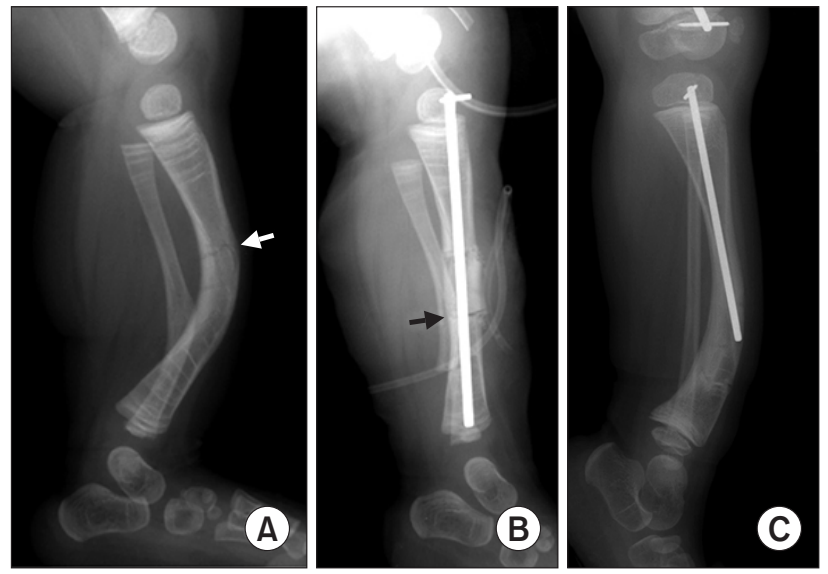

Fig. 4. (A) A 2.5-year-old girl with type III osteogenesis imperfecta sustained a linear fracture (white arrow) at the anteriorly angulated left tibia. (B) Additional osteotomy (black arrow) was performed to straighten the tibia, which was fixed with a non-elongating intramedullary rod because the tibia was too small to fit a telescopic rod. (C) Two years later, the tibia outgrew the rod and angulated at its distal tip. because it facilitates early mobilization and prolonged protection. The authors do not hesitate to perform surgical fixation for a femoral or tibial shaft fracture after 3 years of age. Preexisting angular deformity predisposes a long bone to recurrent fractures. Hence, if surgical fixation is attempted, the preexisting deformity should be corrected simultaneously (Fig. 4A and B).

Femoral neck fractures are uncommon. ${ }^{30)}$ They may be difficult to detect because the onset may be insidious in undisplaced or delayed-malunion type fractures, which may be associated with pain while walking. Although a preexisting IM rod is a challenge for screw fixation, screw could be inserted by passing behind or in front of the IM rod in most cases with special attention to the 3-dimensional perception (Fig. 5).

\section{MANAGEMENT OF LONG BONE DEFORMITY AND PSEUDARTHROSIS}

Moderate-to-severe OI long bones tend to undergo angular deformation with or without a fracture, even when they harbor IM rods. The tibia tends to develop anterolateral bowing, while the femur anterior/varus angulation and/ or retroversion, and the humerus varus angulation and/or internal rotation deformity. If the long bone deformity is so severe that it interferes with limb function or adjacent joint motion, an elective surgery for deformity correction may be indicated to straighten the long bone.

Femoral retroversion may develop gradually, but frequently during fracture/osteotomy healing process, because the IM rod does not provide rotational stability. Hence, after IM rod fixation for a femoral fracture or osteotomy in children, the limb should be immobilized with the hip in internal rotation position. The authors usually apply an extended long leg splint on the operated side and an ordinary long leg splint on the contralateral side with knees moderately flexed, and both are connected by a wooden bar to maintain the operated hip in a neutral or internal rotation position. If only a long leg splint is used for the operated limb, it tends to fall aside laterally and keep the hip joint in an external rotation position, caus-
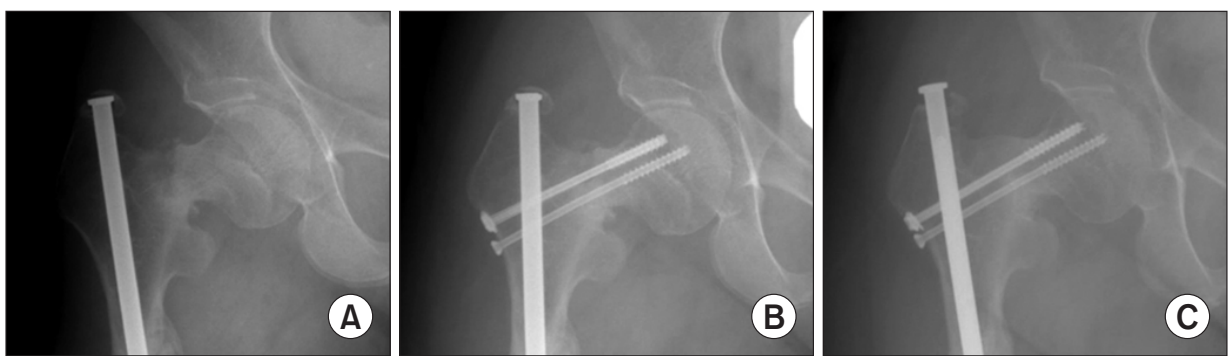

Fig. 5. (A) A 22-year-old woman with type IV OI showed delayed malunion of basicervical stress fracture. (B) Threaded screws were inserted and passed anterior to the preexisting telescoping rod. (C) Bony union was confirmed radiographically at the 1-year follow-up. 
ing retroversion malunion. Retroversion deformity of the femur (Fig. 6) may result in disability due to the marked out-toeing gait, and inability to sit with legs hanging down parallel to each other.

Severely affected patients with inadequate management of fractures may develop pseudarthrosis of a long bone, commonly at the distal humerus, and the proximal or distal femur. It may not be painful but significantly disabling because it precludes weight-bearing of the lower extremity and forceful motion of the upper extremity. It should be managed via rigid fixation and autogenous bone graft, both of which are not easy to perform in patients with OI. Additional fixation that augments the IM rod and an allograft sandwich technique may be helpful. Mobility of the adjacent joint is a prerequisite for osteosynthesis. Long-term persistence of pseudarthrosis may lead to stiffening of an adjacent joint (Fig. 7) because all the motion occurs at the pseudarthrosis site. A stiff adjacent joint prevents bony union, which even if achieved is disabling because the only mobile segment in that area is lost.

Generally, fewer fractures are associated with the upper extremity than the lower extremity but its alignment and stabilization are required for daily self-care and to use walking aids such as crutches or drive a wheelchair. Significant angular deformity interferes with the adjacent joint motion. As a result, the 3-dimensional space within the reach of hand may shrink, causing limitation of daily living (Fig. 8A). Because of the anatomical configuration of the humerus, radius, and ulna, it is impossible to install a telescopic rod anchoring both proximal and distal epiphyses as in the lower extremity. Hence, the authors usually use a non-telescopic rod such as Kirschner wire,
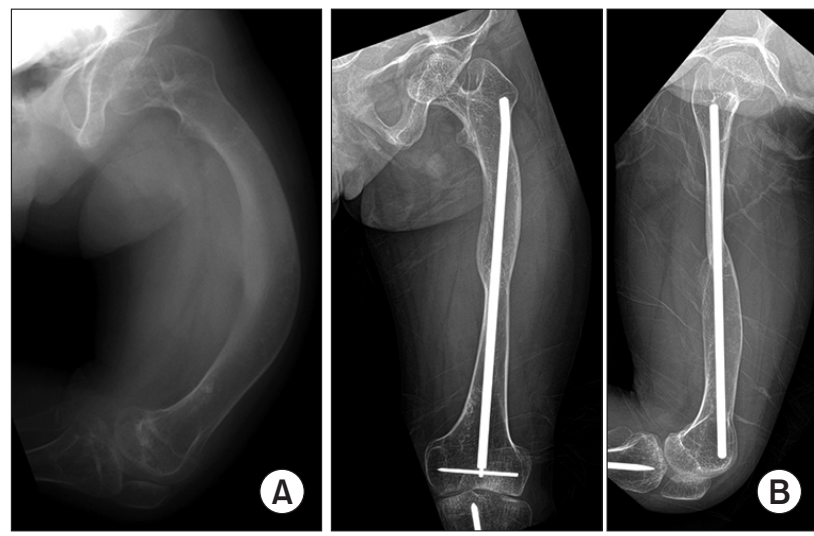

Fig. 6. (A) A 19-year-old woman with type III OI manifested approximately $90^{\circ}$ retroversion of the femur. The proximal femur appeared to be an anteroposterior (AP) projection, but the distal femur to be a lateral projection. (B) After extension and rotational osteotomy, the femur showed normal configuration on both AP and lateral projections.
Steinmann pin, or flexible IM rod for fixation in the upper extremity.

\section{FIXATION OPTIONS FOR FRACTURE AND/OR OSTEOTOMY}

Special considerations are needed for the selection of a fixator for fracture or osteotomy in OI patients. A fixator for OI patients should minimize stress riser effect and last life

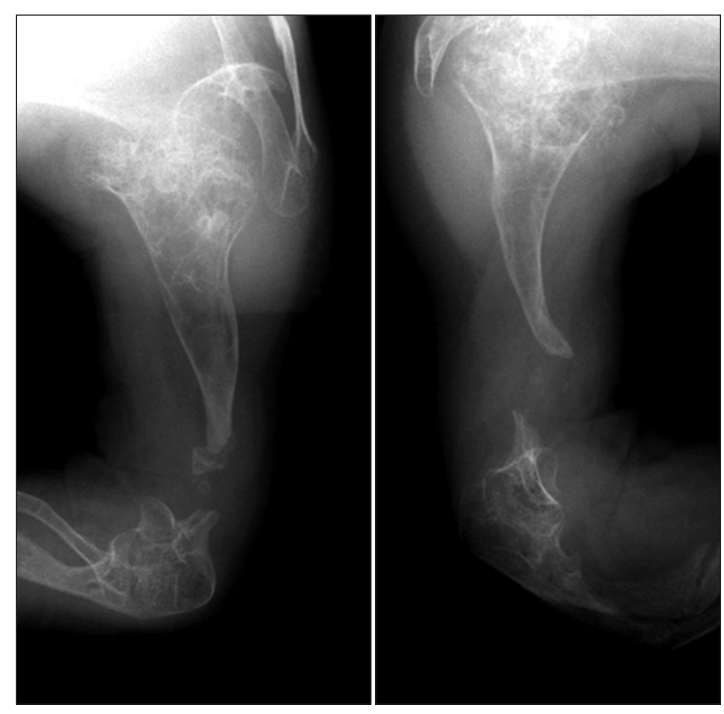

Fig. 7. Pseudarthrosis of the distal humerus in a 19-year-old man, who grew up in an orphanage. The elbow joint was stiff and all the motion occurred at the pseudarthrosis site, so the lesion was skillfully neglected.

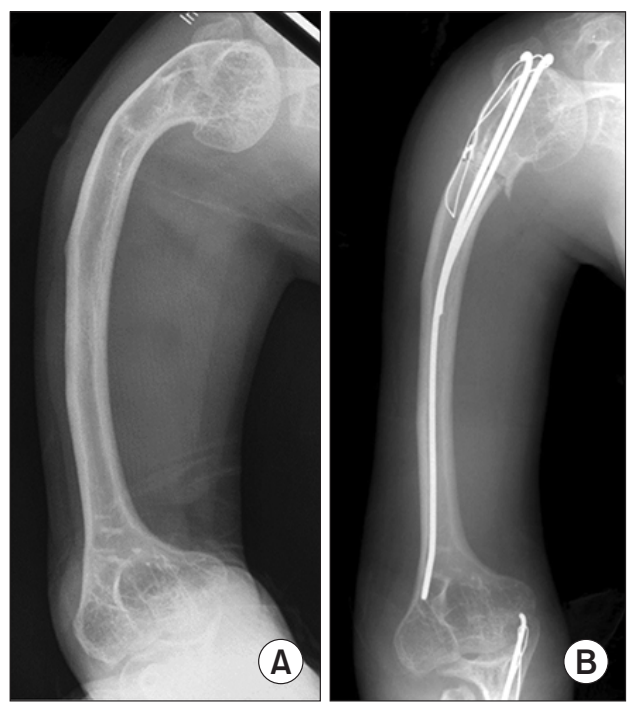

Fig. 8. Proximal humerus varus deformity in a 30 -year-old woman with type III osteogenesis imperfecta $(A)$ was corrected by closing wedge osteotomy fixed with tension band wiring (B). Two long flexible intramedullary rods were used to avoid a stress-riser effect. 
long or possibly until skeletal maturity. A plate and screws, whether dynamic compression or locking style, may lead to effective bony union of the fracture or osteotomy site, but is associated with a high risk of stress concentration at the tip of plate and subsequent peri-implant fractures (Fig. 9). Therefore, its use should be limited to conditions in which other methods cannot be used. Inserting a single or multiple screws without plate will also elevate the stress and should be avoided if possible.

Tension-bands are useful for the fixation of a small fragment at the end of long bones. Fixation of olecranon fractures or metaphyseal osteotomy (Fig. 8) are good candidates for this kind of fixation. In order to avoid a stress riser effect at the tip of pins, long IM pins covering the whole length of long bone, is preferred if possible.

The IM rod is the fixation of choice for long bones in OI patients because it is not complicated with the stress riser effect and protects the whole length of a long bone. Moreover, it does not shield the stress from the bone tissue it protects, preventing osteopenia. However, a flexible IM rod based on a 3-point fixation principle may be associated with complications in OI patients. A tip of flexible rod, placed within the IM cavity, may easily cut out through the osteopenic cortex, irritate the overlying soft tissue, and make a weak point (Fig. 10). On the other hand, a tip placed out of the cortex for later removal may migrate out to irritate the overlying soft tissue or cut through into the medullary cavity, making its removal difficult or impossible. Hence, the use of flexible IM rods in OI patients should be limited only to a long bone with compensatory thickening of the cortex when the surgeon decides to fix a
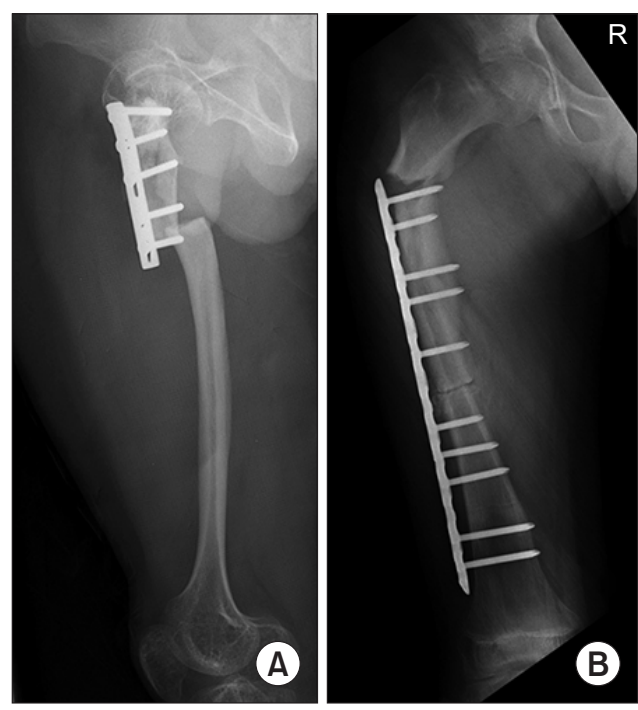

Fig. 9. Two cases of peri-implant fractures at the tip of plate screw: a 30-year-old man (A) and a 14-year-old boy (B). curved long bone without straightening it (Fig. 8B).

A rigid, straight IM rod is the best choice for OI long bone fixation. The long bone should be straightened to harbor this kind of device, and it makes the bone mechanically sounder. Because the long bones of OI patients suffering from multiple fractures usually carry diffuse and/or multifocal angular or torsional deformities, they can be straightened by multi-level osteotomies. Sofield procedure of multiple osteotomy and IM rod fixation ${ }^{31)}$ is the gold standard of treatment for long bone stabilization in an OI patient. A straightened long bone harboring an IM rod is mechanically stronger, resistant to fracture, and more functional for limb motion. The IM rod also prevents significant displacement of fracture fragments, when a fracture occurs.

In most cases, the IM rod fixation is performed in the growing age. A simple, non-telescopic IM rod is outgrown by the growing long bone sooner or later, which locates the IM rod tip at the metaphysis or diaphysis, inducing the stress riser effect. In moderate to severe OI patients, a fracture or gradual angulation easily develops at that site (Fig. 4C). In order to prevent this complication, a telescopic rod was devised by Bailey and Dubow back in $1963 .^{32)}$ It was well-documented that the elongating or telescopic rod showed significantly better survival than the non-telescopic rod. ${ }^{33)}$ However, the original Bailey-Dubow rod encountered various complications either from the device design or from the abnormal bone tissue. Sheffield rod, a modification of Bailey-Dubow rod, improved the outcome by eliminating the device-oriented complications; ${ }^{34)}$ however, it was still traumatic to install, especially
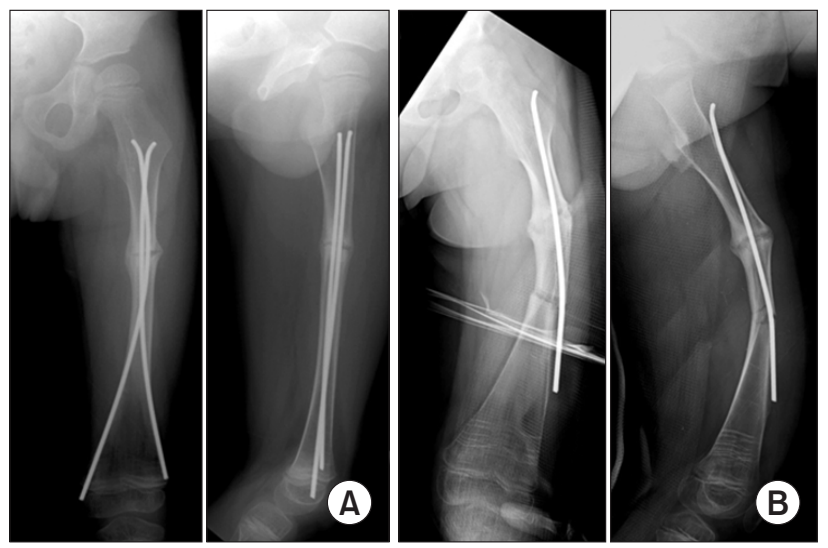

Fig. 10. (A) A 4-year-old boy was referred after his femoral shaft fracture was fixed with flexible intramedullary rods. (B) One rod was removed as it migrated distally. The remaining rod cut through the anterolateral cortex in both proximal and distal areas. The protruding tip irritated the overlying muscles, and eventually a fracture occurred at the distal cutthrough point. 


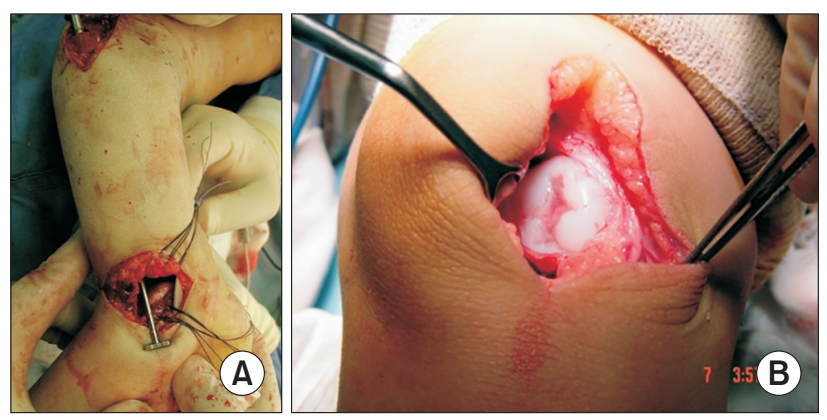

Fig. 11. (A) Implantation of a Sheffield rod into the tibia. The T-piece of an obturator was introduced into the distal tibial epiphysis via ankle arthrotomy and through the articular cartilage. (B) Repeat arthrotomy of the knee joint showed the scar at the distal femoral articular cartilage following implantation of the obturator of Sheffield rod.
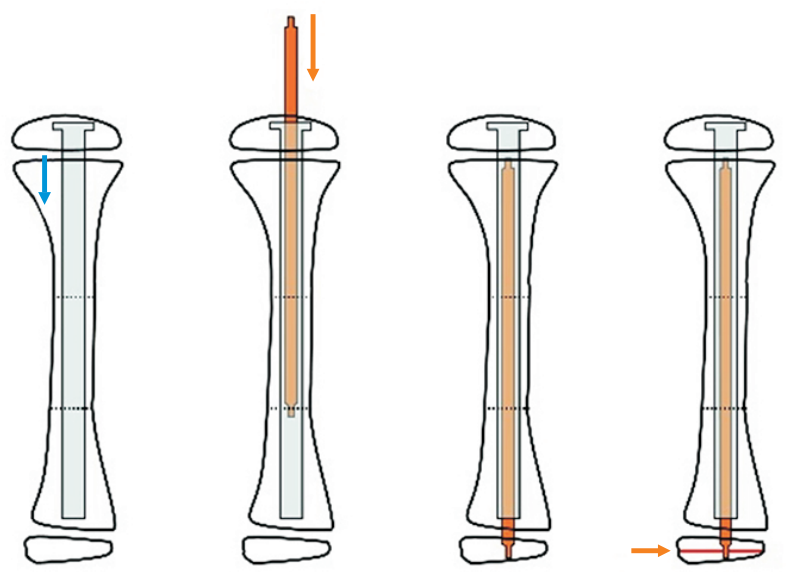

Fig. 12. Schematic drawing of the surgical procedure for insertion of an interlocking telescopic rod in the tibia. The obturator is inserted within the sleeve in an antegrade manner and is transfixed at the distal epiphysis. Adapted from Cho et al. ${ }^{35)}$ with permission of Wolters Kluwer Health. to the distal femur and distal tibia (Fig. 11). Then, the next-generation telescopic rods that facilitate antegrade insertion of the obturator without arthrotomy of the distal joint and without damage to the distal articular cartilage of the long bone were introduced, including the interlocking telescopic rod, ${ }^{35,36)}$ Fassier-Duval rod, ${ }^{37)}$ and corkscrew tipped telescopic $\operatorname{rod}^{38)}$ (Figs. 12 and 13). The basic design of the telescopic rod is a combination of a hollow sleeve and its obturator. The proximal end of a sleeve is anchored at the proximal epiphysis while the distal end of an obturator at the distal epiphysis. The original anchorage mechanism was T-piece in both Bailey-Dubow rod and Sheffield rod, which evolved into an interlocking pin, a threaded end of the rod, or cork-screw end (Fig. 14). If the rod fails to telescope for any reason, either distal or proximal anchorage system fails eventually, and the long bone physis grows over it. Radiographically, the anchorage system of the rod is located at the metaphysis. The rod may appear to have migrated into the medullary space; however, actually the physis of long bone grew over the anchorage system (Fig. 15). No matter how perfect a long bone is straightened and a telescopic rod is placed at its center, a weak long bone of a severely affected patient may spontaneously and gradually angulate even with the IM rod in situ, resulting in the migration of the anchoring system and cutting through the cortex.

Generally, an external fixator is not appropriate for long bone fixation in a patient with OI, because the most proximal or most distal pin becomes a stress riser, and its fixing pins tend to lose rapidly in OI patients. The correction of severe angular deformity of the long bone may occasionally require gradual correction using an external fixator to diminish the risk of neurovascular complication. ${ }^{39)}$ However, the use of the external fixator should be
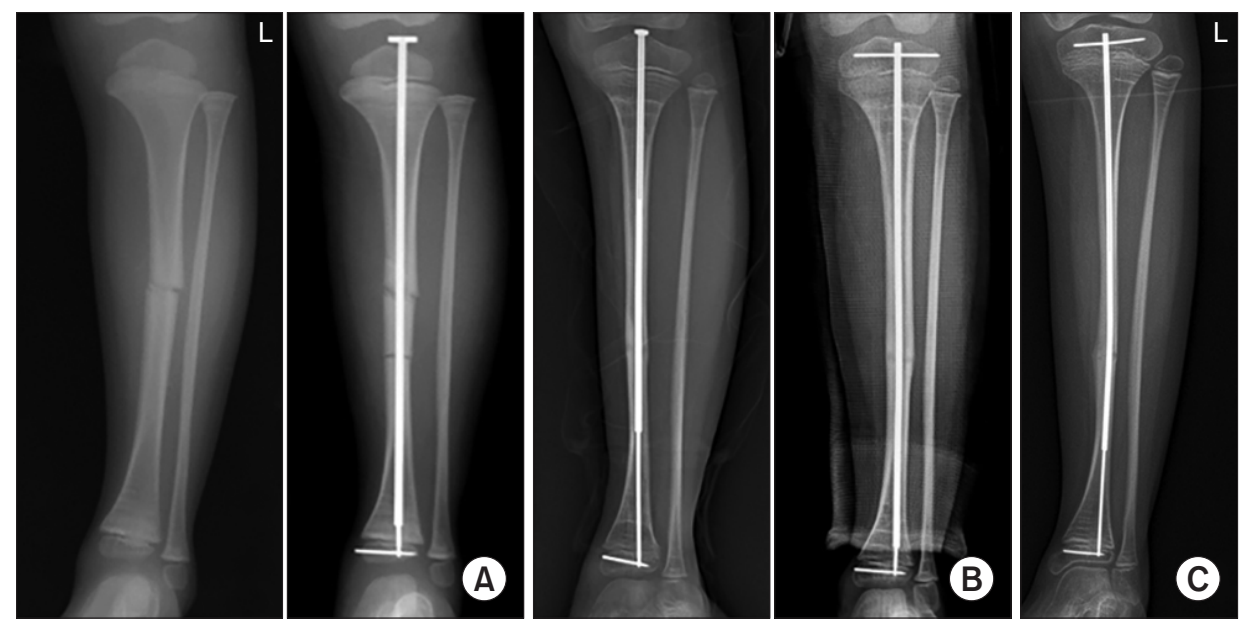

Fig. 13. (A) A 5-year-old boy with type IV osteogenesis imperfecta sustained a tibial shaft fracture and was treated with open reduction-additional osteotomyinterlocking telescopic intramedullary rod fixation. (B) Four years later, the female rod was replaced with a longer dual interlocking telescopic rod. (C) Until age 13 years, the rod telescoped successfully and continued to stabilize the tibia. 

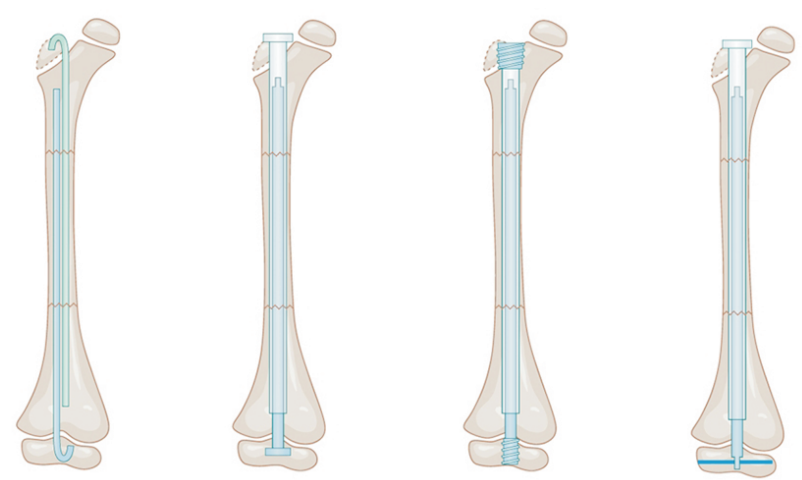

Fig. 14. Telescopic rod designs. (From left) Dual Rush pins; Sheffield rod; Fassier-Duval rod; and interlocking telescopic rod of the authors' design.
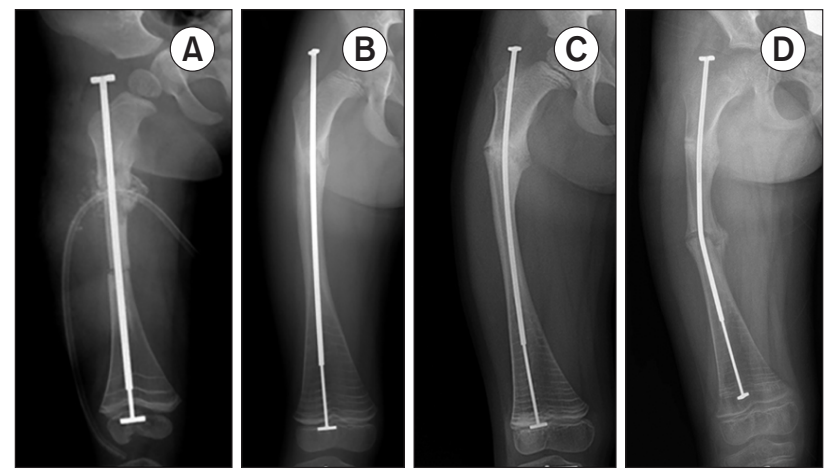

Fig. 15. (A) A 2-year-old girl with type IV osteogenesis imperfecta underwent Sofield operation on the right femur, which was fixed with a Sheffield rod. (B) The rod telescoped successfully for 3 years. (C) At age 7 years, the rod was bent at the proximal femur and failed to telescope. The T-piece of the obturator was located at the physis. (D) At the age of 8 years, the rod was further bent, and the T-piece of the obturator was located at the distal metaphysis.
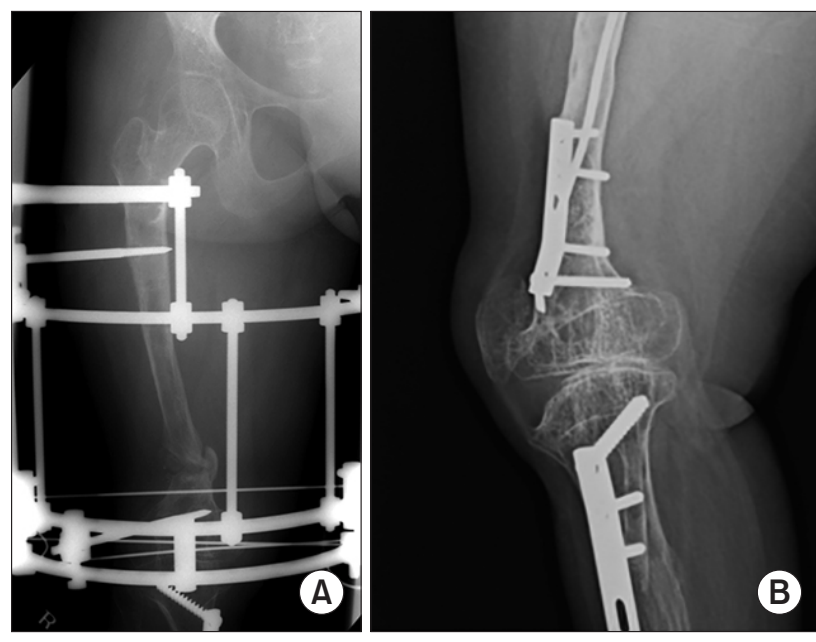

Fig. 16. (A) A 24-year-old woman with type IV osteogenesis imperfecta was referred due to nonunion of a distraction gap during femoral lengthening for height. (B) The distal femoral condyle collapsed, resulting in total stiff knee and severe disability in the patient. minimized and converted to an IM rod as soon as possible. Patients with OI are more tolerable to soft-tissue stretching than normal population, so acute correction of a large angular deformity and internal fixation using an IM rod is better tolerated than in normal population. Another option is to combine shortening of the long bone with acute correction of large angular deformity. Limb lengthening using an external fixator was theoretically possible in patients with type I OI, and a successful result was reported. ${ }^{40)}$ However, the benefit should be weighed against the risk of poor regenerate bone formation, development of deformity at the regenerated bone after external fixator removal, and most importantly, adjacent joint disfiguration due to increased joint pressure (Fig. 16).

\section{AUGMENTING FIXATION}

Generally, OI patients do not manifest poor bone healing capacity for their age. However, poor bone stock in adolescents or adults with OI interferes with effective fixation and bone healing mechanism. With age, the diaphysis of long bone in moderate-to-severe OI patients tends to be relatively thin for its length or for the body size. Hence, only the IM rod fitting the thin diaphysis is very thin for the long bone or the body size, which cannot provide sufficient fixation for fracture/osteotomy healing. Moreover, continuous administration of bisphosphonate suppresses bone remodeling, which is a critical final step in bone healing process. Failure to achieve complete bony union or development of a cortical crack, which does not heal completely, is not uncommon in adolescent or adult patients with OI. Additional fixation to augment IM rodding may be needed for fracture treatment, osteotomy fixation, or osteosynthesis of delayed or nonunion of the OI long bone, especially in adolescents and adults. Although the IM space is already occupied by an IM rod, a locking plate and unicortical screw fixation can provide additional fixation (Fig. 17). This technique was made possible with the advent of a locking plate design. If the diaphyseal cortex is too thin, a longer plate should be selected that could purchase the metaphysis on both sides. Because the plate and screws are also a stress-riser, they should be removed as soon as union is achieved. Another option to augment fixation of long bone in OI cases with poor bone stock is the so-called sandwich technique, in which cortical allograft straps are applied to the circumference of the lesion. ${ }^{41)} \mathrm{We}$ have only early and limited experience of this technique. Even with the risk of infection and devitalization of the adjacent bone segments, augmentation of bone stock, when it is desperately needed, is highly beneficial. 

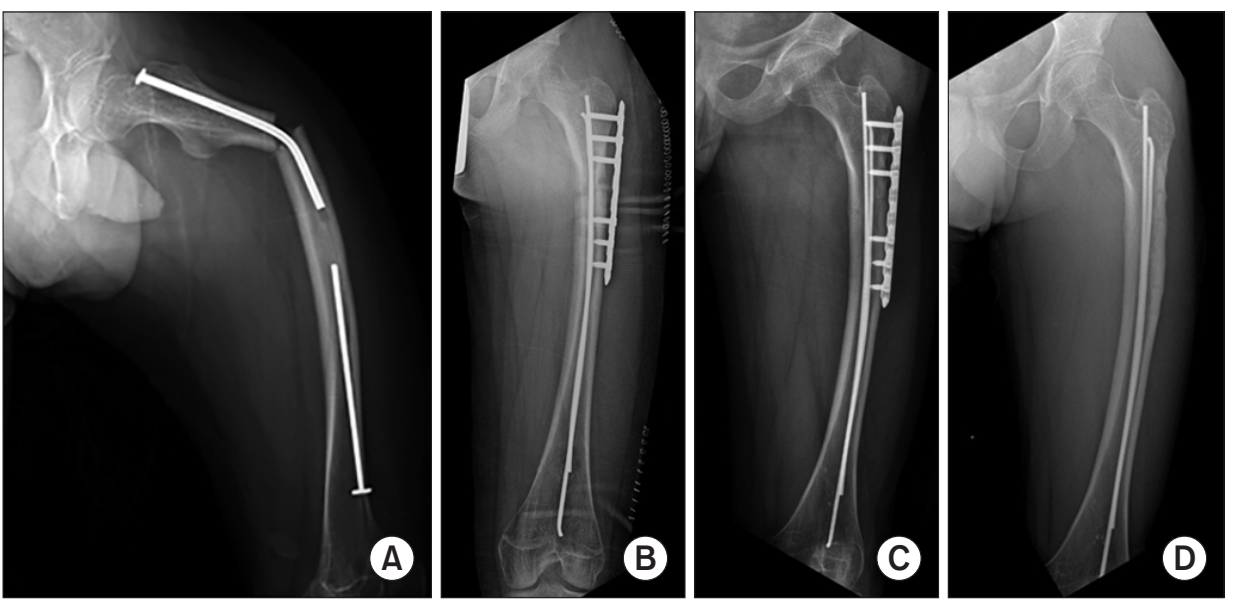

Fig. 17. (A) A 16-year-old boy with type I osteogenesis imperfecta sustained a subtrochanteric femur fracture. (B) Open reduction and internal fixation with 2 small flexible intramedullary (IM) rods and a locking plate with unicortical screws. (C) Bony union was achieved in 3 years, and the plate and screws were removed. (D) The IM rods were left behind by the age of 21 years.

\section{SPINE STABILIZATION}

Scoliosis is observed in $39 \%$ to $80 \%$ of patients with OI: $39 \%$ in type I, $68 \%$ in type III, and $54 \%$ in type IV cases. Lower DEXA Z-score is known to be related to the development and progression of scoliosis. Theoretically, the etiology of scoliosis may involve shape and fragility of vertebral body, ligamentous laxity and muscle weakness, limb length discrepancy, and pelvic obliquity. The most common type of curvature is the single thoracic curve, which is observed in $97 \%$ of scoliosis in patients with type I OI and $58 \%$ in those with type III OI. Scoliosis in patients with OI is known to progress even in adulthood: $6^{\circ}$ annually in type III, $4^{\circ}$ in type IV, and $1^{\circ}$ in type I. In general, bisphosphonate treatment for OI patients has proved no beneficial effect on the development and progression of scoliosis. However, Anissipour et al. ${ }^{17)}$ reported that bisphosphonate treatment in type III OI patients before age 6 years can slow down annual progression of scoliosis down to $2.3^{\circ}$ from $4^{\circ}$. Progression of scoliosis leads to restrictive pulmonary disease, and the predicted vital capacity is reduced to $41 \%$ in patients with larger than $60^{\circ}$ thoracic scoliosis.

Fragile bone and rigid deformity are challenges for the treatment of scoliosis in patients with OI. Hard braces such as a Milwaukee brace aggravates rib deformity and may induce a fracture. Although a soft brace plays a limited role in patients adopting a sitting posture, it cannot prevent progression of deformity. Typically with progressive deformity larger than $35^{\circ}$ to $45^{\circ}$ and age above 8 years, surgical spine fusion may stop the progression of deformity. For treatment of patients with earlier progression of scoliosis, especially before the age of 4 years, the possible development of thoracic insufficiency syndrome should be considered. Classically surgical complications are reported in more than $50 \%$, especially in patients with intraoperative bleeding and instrumental problems, such
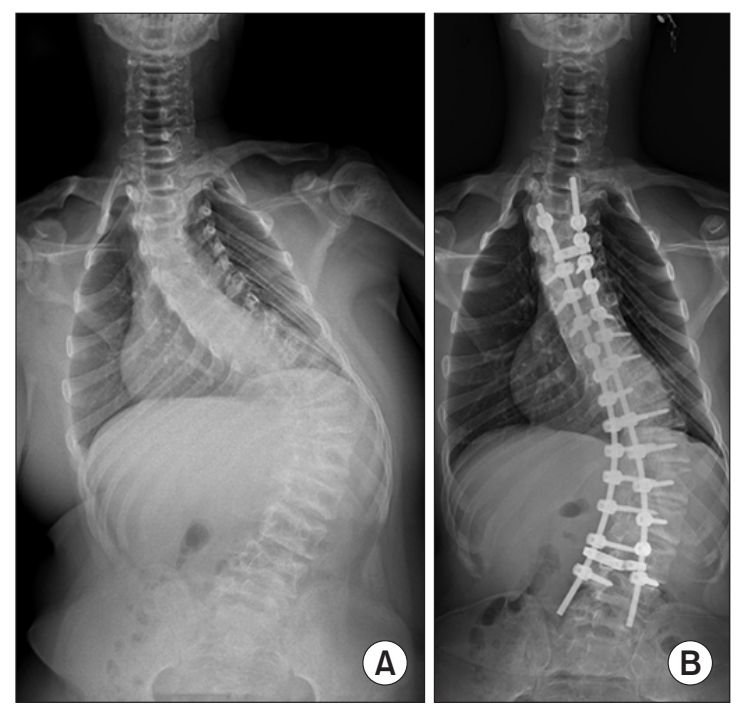

Fig. 18. (A) A 13-year-old boy with type $V$ osteogenesis imperfecta underwent posterior spinal fusion with modern pedicle screw-based instrument for treatment of progressive scoliosis. (B) Five years later, a postoperative radiograph showed adequate spinal balance without further progression of deformity.

as loss of fixation. Recent surgical advances with modern pedicle screw-based instruments are expected to provide better fixation and correction outcomes with a lower rate of surgical complications (Fig. 18). All segmental pedicle screw fixation can minimize mechanical stress on each fixation point during corrective maneuvers and Ponte osteotomy or even 3-column osteotomy can be used to help increase flexibility of the rigid deformity. Some authors prefer to apply preoperative skeletal traction to achieve better correction for rigid and advanced deformity, ${ }^{42)}$ and some advocate to use polymethyl methacrylate augmentation to increase the purchasing power of pedicle screws. ${ }^{43)}$ With all other techniques, the operator should set a practi- 
cal goal of deformity correction, as an excessive corrective force on the rigid deformity may lead to loss of fixation.

Other serious spinal problems to which clinicians should pay attention in OI patients is craniovertebral junction (CVJ) anomaly. Up to $26 \%$ to $37 \%$ of patients with OI show CVJ anomaly, including basilar invagination, impression, and platybasia in $4 \%$ to $13 \%, 6 \%$ to $25 \%$, and $16 \%$ to $29 \%$, respectively. Basilar invagination is protrusion of dens into the foramen magnum, and basilar impression is defined as lowering of the cranial base (occipital condyles and foramen magnum). Platybasia involves flattening of the cranial base. All these CVJ abnormalities can cause neurological impairment, and neurological symptoms have been reported in $8 \%$ of all patients with OI. Before the age of 6 years, patients with OI are recommended to undergo baseline and serial follow-up lateral skull or cervical spine radiographs as well as whole spine X-rays. A baseline MRI of CVJ is recommended to facilitate future clinical decisions in terms of progress of disease. During the follow-up of OI patients, clinicians should pay attention to headaches, symptoms related to cranial nerve palsy, dysphagia, and symptoms and signs of myelopathy, such as hyperreflexia, quadriparesis, and gait abnormality. Asymptomatic patients can be serially and carefully monitored; however, some experts advocate prophylactic treatment even for asymptomatic patients due to the progressive natural course of basilar invagination leading to advanced deformity and dangerous neurologic dysfunction. The authors recommend treatment of patients manifesting such symptoms related to CVJ and in case of radiographic progression of deformity. The treatment goals include reduction and stabilization of bony structures with and without decompression of neural tissues. Closed reduction and stabilization, such as skeletal traction and halo-vest application, or surgical reduction/decompression and internal fixation via posterior or anterior approach can be used for treatment. $^{44)}$

\section{CONCLUSION}

OI includes a wide spectrum of genetic disorders manifesting various severity in bone fragility, as well as spine and limb deformities. More than 15 causative genes have been identified, and gene panel testing using next generation sequencing technology can provide inexpensive genetic diagnosis of OI. A multidisciplinary approach is essential for the management of OI including genetic analysis and counseling, medical treatment, rehabilitation and orthosis application, and surgical intervention. Telescopic rodding and bisphosphonate treatment represent critical breakthroughs in the management of OI patients. Ongoing studies will open new vista for the management including medical, rehabilitation, and surgical treatments.

\section{CONFLICT OF INTEREST}

No potential conflict of interest relevant to this article was reported.

\section{ACKNOWLEDGEMENTS}

This research was supported by Genome Technology to Business Translation Program of the National Research Foundation (NRF) funded by the Ministry of Science, ICT \& Future Planning (NRF-2014M3C9A2064684).

\section{REFERENCES}

1. Cho TJ, Lee KE, Lee SK, et al. A single recurrent mutation in the 5'-UTR of IFITM5 causes osteogenesis imperfecta type V. Am J Hum Genet. 2012;91(2):343-8.

2. Semler O, Garbes L, Keupp K, et al. A mutation in the 5'UTR of IFITM5 creates an in-frame start codon and causes autosomal-dominant osteogenesis imperfecta type $\mathrm{V}$ with hyperplastic callus. Am J Hum Genet. 2012;91(2):349-57.

3. Mortier GR, Cohn DH, Cormier-Daire V, et al. Nosology and classification of genetic skeletal disorders: 2019 revision. Am J Med Genet A. 2019;179(12):2393-419.

4. Keupp K, Beleggia F, Kayserili H, et al. Mutations in WNT1 cause different forms of bone fragility. Am J Hum Genet. 2013;92(4):565-74.
5. Trejo P, Palomo T, Montpetit K, et al. Long-term followup in osteogenesis imperfecta type VI. Osteoporos Int. 2017;28(10):2975-83.

6. Smith R, Francis MJ, Houghton GR. The Brittle bone syndrome: osteogenesis imperfecta. London: Butterworths; 1983.

7. Sillence DO, Senn A, Danks DM. Genetic heterogeneity in osteogenesis imperfecta. J Med Genet. 1979;16(2):101-16.

8. Glorieux FH, Rauch F, Plotkin H, et al. Type V osteogenesis imperfecta: a new form of brittle bone disease. J Bone Miner Res. 2000;15(9):1650-8.

9. Lee DY, Cho TJ, Choi IH, et al. Clinical and radiological 
Cho et al. Osteogenesis Imperfecta

Clinics in Orthopedic Surgery • Vol. 12, No. 4, $2020 \bullet$ www.ecios.org

manifestations of osteogenesis imperfecta type V. J Korean Med Sci. 2006;21(4):709-14.

10. Kim OH, Jin DK, Kosaki K, et al. Osteogenesis imperfecta type V: clinical and radiographic manifestations in mutation confirmed patients. Am J Med Genet A. 2013;161(8):19729.

11. Marini JC, Forlino A, Bachinger HP, et al. Osteogenesis imperfecta. Nat Rev Dis Primers. 2017;3:17052.

12. Chagas CE, Roque JP, Santarosa Emo Peters B, LazarettiCastro M, Martini LA. Do patients with osteogenesis imperfecta need individualized nutritional support? Nutrition. 2012;28(2):138-42.

13. Glorieux FH, Bishop NJ, Plotkin H, Chabot G, Lanoue G, Travers R. Cyclic administration of pamidronate in children with severe osteogenesis imperfecta. N Engl J Med. 1998;339(14):947-52.

14. Dwan K, Phillipi CA, Steiner RD, Basel D. Bisphosphonate therapy for osteogenesis imperfecta. Cochrane Database Syst Rev. 2016;10(10):CD005088.

15. Sato A, Ouellet J, Muneta T, Glorieux FH, Rauch F. Scoliosis in osteogenesis imperfecta caused by COL1A1/COL1A2 mutations: genotype-phenotype correlations and effect of bisphosphonate treatment. Bone. 2016;86:53-7.

16. Trejo P, Rauch F. Osteogenesis imperfecta in children and adolescents-new developments in diagnosis and treatment. Osteoporos Int. 2016;27(12):3427-37.

17. Anissipour AK, Hammerberg KW, Caudill A, et al. Behavior of scoliosis during growth in children with osteogenesis imperfecta. J Bone Joint Surg Am. 2014;96(3):237-43.

18. Sakkers R, Kok D, Engelbert R, et al. Skeletal effects and functional outcome with olpadronate in children with osteogenesis imperfecta: a 2-year randomised placebocontrolled study. Lancet. 2004;363(9419):1427-31.

19. Anam EA, Rauch F, Glorieux FH, Fassier F, Hamdy R. Osteotomy healing in children with osteogenesis imperfecta receiving bisphosphonate treatment. J Bone Miner Res. 2015;30(8):1362-8.

20. Bargman R, Posham R, Boskey AL, DiCarlo E, Raggio C, Pleshko N. Comparable outcomes in fracture reduction and bone properties with RANKL inhibition and alendronate treatment in a mouse model of osteogenesis imperfecta. Osteoporos Int. 2012;23(3):1141-50.

21. Sinder BP, White LE, Salemi JD, et al. Adult Brtl/+ mouse model of osteogenesis imperfecta demonstrates anabolic response to sclerostin antibody treatment with increased bone mass and strength. Osteoporos Int. 2014;25(8):2097-107.

22. Gatti D, Rossini M, Viapiana O, et al. Teriparatide treatment in adult patients with osteogenesis imperfecta type I. Calcif
Tissue Int. 2013;93(5):448-52.

23. Baylor College of Medicine. Safety of fresolimumab in the treatment of osteogenesis imperfecta. Bethesda: U.S. National Library of Medicine; 2020.

24. Antoniazzi F, Monti E, Venturi G, et al. GH in combination with bisphosphonate treatment in osteogenesis imperfecta. Eur J Endocrinol. 2010;163(3):479-87.

25. Horwitz EM, Prockop DJ, Gordon PL, et al. Clinical responses to bone marrow transplantation in children with severe osteogenesis imperfecta. Blood. 2001;97(5):1227-31.

26. Franzone JM, Shah SA, Wallace MJ, Kruse RW. Osteogenesis imperfecta: a pediatric orthopedic perspective. Orthop Clin North Am. 2019;50(2):193-209.

27. Veilleux LN, Pouliot-Laforte A, Lemay M, Cheung MS, Glorieux $\mathrm{FH}$, Rauch $\mathrm{F}$. The functional muscle-bone unit in patients with osteogenesis imperfecta type I. Bone. 2015;79:527.

28. Mueller B, Engelbert R, Baratta-Ziska F, et al. Consensus statement on physical rehabilitation in children and adolescents with osteogenesis imperfecta. Orphanet J Rare Dis. 2018;13(1):158.

29. Marr C, Seasman A, Bishop N. Managing the patient with osteogenesis imperfecta: a multidisciplinary approach. J Multidiscip Healthc. 2017;10:145-55.

30. Chow W, Negandhi R, Kuong E, To M. Management pitfalls of fractured neck of femur in osteogenesis imperfecta. J Child Orthop. 2013;7(3):195-203.

31. Sofield HA, Millar EA. Fragmentation, realignment, and intramedullary rod fixation of deformities of the long bones in children: a ten-year appraisal. J Bone Joint Surg Am. 1959;41(8):1371-91.

32. Bailey RW, Dubow HI. Studies of longitudinal bone growth resulting in an extensible nail. Surg Forum. 1963;14:455-8.

33. Marafioti RL, Westin GW. Elongating intramedullary rods in the treatment of osteogenesis imperfecta. J Bone Joint Surg Am. 1977;59(4):467-72.

34. Wilkinson JM, Scott BW, Clarke AM, Bell MJ. Surgical stabilisation of the lower limb in osteogenesis imperfecta using the Sheffield Telescopic Intramedullary Rod System. J Bone Joint Surg Br. 1998;80(6):999-1004.

35. Cho TJ, Choi IH, Chung CY, Yoo WJ, Lee KS, Lee DY. Interlocking telescopic rod for patients with osteogenesis imperfecta. J Bone Joint Surg Am. 2007;89(5):1028-35.

36. Shin CH, Lee DJ, Yoo WJ, Choi IH, Cho TJ. Dual interlocking telescopic rod provides effective tibial stabilization in children with osteogenesis imperfecta. Clin Orthop Relat Res. 2018;476(11):2238-46. 
37. Fassier F. Fassier-Duval telescopic system: how i do it? J Pediatr Orthop. 2017;37 Suppl 2:S48-S51.

38. Sarikaya I, Seker A, Erdal OA, Gunay H, Inan M, Guler B. Using a corkscrew-tipped telescopic nail in the treatment of osteogenesis imperfecta: a biomechanical study and preliminary results of 17 consecutive cases. J Pediatr Orthop B. 2019;28(2):173-8.

39. To M, Gupta V, Chow W. Surgical management of long bone pseudarthrosis with severe limb length discrepancy in osteogenesis imperfecta. J Pediatr Orthop B. 2013;22(1):639.

40. Saldanha KA, Saleh M, Bell MJ, Fernandes JA. Limb lengthening and correction of deformity in the lower limbs of children with osteogenesis imperfecta. J Bone Joint Surg Br. 2004;86(2):259-65.
41. Puvanesarajah V, Shapiro JR, Sponseller PD. Sandwich allografts for long-bone nonunions in patients with osteogenesis imperfecta: a retrospective study. J Bone Joint Surg Am. 2015;97(4):318-25.

42. Wallace MJ, Kruse RW, Shah SA. The spine in patients with osteogenesis imperfecta. J Am Acad Orthop Surg. 2017; 25(2):100-9.

43. O’Donnell C, Bloch N, Michael N, Erickson M, Garg S. Management of scoliosis in children with osteogenesis imperfecta. JBJS Rev. 2017;5(7):e8.

44. Ibrahim AG, Crockard HA. Basilar impression and osteogenesis imperfecta: a 21-year retrospective review of outcomes in 20 patients. J Neurosurg Spine. 2007;7(6):594-600. 